\title{
16. INTERSTITIAL WATER CHEMISTRY, LEG 16
}

\author{
B. J.Presley, Department of Oceanography, Texan A\&M University, College Station, Texas \\ and \\ Chari Petrowski and I. R. Kaplan, Department of Geology and Institute of Geophysics and Planetary Physics, \\ University of California, Los Angeles, California
}

\section{INTRODUCTION AND PROCEDURES}

Leg 16 of the Glomar Challenger began in San Cristobal, Panama, and ended in Honolulu, Hawaii. Nine drilling sites were occupied along the way and pore water was collected at seven of these. The most extensive pore water sampling was done at Sites 157 and 158 which are in the Panama Basin area, just off the coast of South and Central America. The lithology at these sites was fairly uniform chalk ooze and marl with various admixtures of volcanic ash and pyrite down to an extrusive basalt basement.

The other pore water samples were collected from sediments taken along the northern edge of the equatorial band of biogenous sediments lying between the Clarion and Clipperton fracture zones. These sediments are somewhat less homogeneous than the nearshore biogenous sediments from the Panama Basin, but are nevertheless very similar to them in most respects. Abundant Fe-Mn oxide material was recovered in many of the samples and while they may be related to the so-called "basal amorphous iron oxide facies" discussed in earlier leg reports dealing with the eastern Pacific, their origin does not appear to be a simple process.

The analytical procedures currently being used in our pore water program are essentially those described in some detail elsewhere (Presley, 1971; Presley and Claypool, 1971). These procedures have evolved as we have sought to get more and better data from the small samples provided by the Deep Sea Drilling Project. Therefore, the procedures used for Leg 16 may, in some instances, be modifications of those described in the references, but any changes are considered not sufficiently significant to warrant a separate description here.

\section{RESULTS AND DISCUSSION}

All data that we have obtained to date from the water samples are given in Table 1, except major cation concentrations which were omitted, as they will be discussed in a later report by Sayles and others. Lithium could not be obtained for these samples due to a malfunction of our flame photometer.

Chloride and bromide values have been close to those of average seawater in samples from previous legs, except in locations where the presence of evaporites was known or implied, and a few locations apparently influenced by continental ground water. The Leg 16 samples show small random variations in both components with depth, but since the variations are only slightly larger than our usual analytical error and no duplicate analyses were possible, no real significance can be attached to them.
The most unusual sample is the bottom one from Site 163 , which was found to be depleted in both $\mathrm{Cl}$ and $\mathrm{Br}$ by about $5 \%$. This is most likely a valid measurement because it agrees with the shipboard salinity determination, but it may have no geochemical significance.

The boron concentration shows a gradual increase with depth at Site 157, so that the bottom sample gives twice the seawater value, but at nearby and lithologically similar Site 158 there is a $25 \%$ decrease in B concentration with depth. The other sites show little variation in B content.

The dissolved $\mathrm{Si}$ concentration is extremely high in all Leg 16 samples, presumably due to the presence of siliceous fossils and volcanic ash. Removal of the water at higher than in situ temperatures may have had a small effect on the $\mathrm{Si}$ concentrations, but since the in situ temperatures are not precisely known, this is difficult to evaluate.

Ammonia concentrations increase to high values at depth in the two nearshore sites, such that on a molar basis $\mathrm{NH}_{3}$ almost equals $\mathrm{CO}_{2}$ at Site 157 and is actually higher at Site 158. This has been noted at many sites on previous legs. The central ocean samples from this leg generally had a measurable $\mathrm{NH}_{3}$ concentration, but one about 10 times less than that in the nearshore samples.

With the exception of Site 157 and the upper samples from Site 158, there was little evidence for excess dissolved bicarbonate. As stated above, these higher values generally correlate with higher ammonia concentrations and must be due to decomposition of organic matter. However, in several samples the value of dissolved $\Sigma \mathrm{CO}_{2}$ dropped below that in normal seawater. In some cases, as in Sample $161-1-2$, it probably resulted from loss of $\mathrm{CO}_{2}$ on board ship prior to sampling. In the case of samples from Site 163 , dissolved carbonate may have been removed by exchange with a solid phase. In a number of samples isotopically light $\delta \mathrm{C} 13$ values were obtained. There is no explanation for the values for Samples 159-1-5 and 163-2-4. It is also difficult to understand why samples depleted in dissolved $\mathrm{CO}_{2}$ should be isotopically light. It may indicate upward migration of methane and oxidation near the sediment-water interface.

\section{ACKNOWLEDGMENTS}

This work was supported in part by AEC Grant AT (11-1)-34 P.A. 134 and NSF Grant GA-20715.

\section{REFERENCES}

Presley, B. J., 1971. Techniques for analyzing interstitial water samples. Part I: Determination of selected minor and major inorganic constituents. In Winterer, E. L., Riedel, W. R. et al., 1971. Initial Reports of the Deep 
Sea Drilling Project, Volume 7. Washington (U.S. Government Printing Office) 1749.

Presley, B. J. and Claypool, G. E., 1971. Techniques for analyzing interstitial water samples. Part III. Determination of total dissolved carbonate and carbon isotope ratios. In Winterer, E. L., Riedel, W. R. et al., 1971.
Initial Reports of the Deep Sea Drilling Project, Volume 7. Washington (U.S. Government Printing Office). 1756.

Presley, B. J. and Kaplan, I. R., 1968. Changes in dissolved sulfate, calcium and carbonate from interstitial water of nearshore sediments. Geochim. Cosmochim. Acta. 32, 1037.

TABLE 1

Selected Major and Minor Constituents and $\delta \mathrm{C}^{\mathbf{1 3}}$, Interstitial Water, Leg 16

\begin{tabular}{|c|c|c|c|c|c|c|c|c|c|c|}
\hline Sample & $\begin{array}{c}\text { Depth }^{\mathrm{a}} \\
\text { (m) }\end{array}$ & Age and Description of Sediment ${ }^{b}$ & $\begin{array}{c}\mathrm{C} 1 \\
(\mathrm{~g} / \mathrm{Kg})\end{array}$ & $\begin{array}{c}\mathrm{Br} \\
(\mathrm{mg} / \mathrm{Kg})\end{array}$ & $\begin{array}{c}\mathrm{B} \\
(\mathrm{mg} / \mathrm{Kg})\end{array}$ & $\begin{array}{c}\mathrm{Si} \\
(\mathrm{mg} / \mathrm{Kg})\end{array}$ & $\begin{array}{c}\mathrm{NH}_{3} \\
(\mathrm{mg} / \mathrm{Kg})\end{array}$ & $\underset{(\mathrm{mg} / \mathrm{Kg})}{\mathrm{Mn}}$ & $\underset{(\mathrm{mM} / \mathrm{Kg})}{\Sigma \mathrm{CO}_{2}}$ & $\begin{array}{l}\delta \mathrm{C}^{13} \\
(\mathrm{PDB})\end{array}$ \\
\hline $157-1-2$ & 10 & Pleistocene chalk ooze (diatoms) & 19.4 & 69 & 5.1 & 18.7 & 5.1 & 0.7 & 3.25 & -4.56 \\
\hline $157-3-1$ & 29 & Pleistocene chalk ooze (diatoms, pyrite, $\mathrm{H}_{2} \mathrm{~S}$ ) & - & - & - & - & - & - & 4.32 & -3.95 \\
\hline $157-5-3$ & 50 & Pleistocene chalk ooze (diatoms, pyrite, $\mathrm{H}_{2} \mathrm{~S}$ ) & 19.5 & 61 & 5.4 & 29.5 & 12.7 & 0.2 & 4.80 & -3.62 \\
\hline $157-7-4$ & 70 & Pleistocene chalk ooze (diatoms, pyrite, $\mathrm{H}_{2} \mathrm{~S}$ ) & - & - & - & - & - & - & 4.47 & -4.02 \\
\hline $157-9-6$ & 90 & Pleistocene chalk ooze (diatoms, pyrite, $\mathrm{H}_{2}^{2} \mathrm{~S}$ ) & 19.9 & 68 & - & 33.7 & 12.5 & 0.2 & 6.59 & -5.93 \\
\hline $157-12-5$ & 115 & Pleistocene chalk ooze (diatoms, pyrite, $\mathrm{H}_{2} \mathrm{~S}$ ) & - & - & - & - & - & - & 6.08 & -4.62 \\
\hline $157-15-5$ & 142 & Pleistocene chalk ooze (diatoms, pyrite, $\mathrm{H}_{2} \mathrm{~S}$ ) & 20.4 & 72 & 6.7 & 36.0 & 14.0 & 0.09 & 4.13 & -6.87 \\
\hline $157-17-6$ & 161 & $\begin{array}{l}\text { Late Pliocene chalk ooze (diatoms, pyrite, } \\
\mathrm{H}_{2} \mathrm{~S} \text { ) }\end{array}$ & - & - & - & - & - & - & 5.91 & -3.03 \\
\hline $157-19-6$ & 180 & $\begin{array}{l}\text { Late Pliocene chalk ooze (diatoms, pyrite, } \\
\mathrm{H}_{2} \mathrm{~S} \text { ) }\end{array}$ & 19.8 & 68 & 6.6 & 37.7 & 17.5 & 0.08 & 5.85 & -2.32 \\
\hline $157-28-5$ & 259 & $\begin{array}{l}\text { Early Pliocene chalk ooze (diatoms, pyrite, } \\
\mathrm{H}_{2} \mathrm{~S} \text { ) }\end{array}$ & - & - & - & - & - & - & 3.70 & -0.85 \\
\hline $157-30-6$ & 278 & $\begin{array}{l}\text { Early Pliocene chalk ooze (diatoms, pyrite, } \\
\mathrm{H}_{2} \mathrm{~S} \text { ) }\end{array}$ & 19.9 & 72 & 8.2 & 43.7 & 17.5 & 0.07 & 3.05 & +0.19 \\
\hline $157-32-6$ & 296 & $\begin{array}{l}\text { Early Pliocene chalk ooze (diatoms, pyrite, } \\
\mathrm{H}_{2} \mathrm{~S} \text { ) }\end{array}$ & - & - & - & - & - & - & 4.67 & -0.36 \\
\hline $157-35-1$ & 316 & $\begin{array}{l}\text { Late Miocene chalk ooze (diatoms, pyrite, } \\
\mathrm{H}_{2} \mathrm{~S} \text { ) }\end{array}$ & 19.2 & 63 & 8.7 & 42.3 & 23.0 & 0.08 & 2.67 & -0.33 \\
\hline $157-39-1$ & 346 & $\begin{array}{l}\text { Late Miocene chalk ooze (diatoms, pyrite, } \\
\mathrm{H}_{2} \mathrm{~S} \text { ) }\end{array}$ & 19.5 & 66 & 9.0 & 36.0 & 29.0 & 0.06 & 1.98 & -0.79 \\
\hline $58-1-3$ & 4 & Pleistocene marly chalk (pyrite, ash) & 18.9 & 63 & 6.4 & 15.9 & 7.8 & 0 . & - & - \\
\hline $158-3-6$ & 17 & Pleistocene marly chalk & - & - & - & - & - & - & 2.67 & - \\
\hline $158-5-3$ & 40 & Pliocene marly chalk (pyrite, ash) & 19.3 & 64 & 4.4 & 21.3 & 16.8 & 0.08 & 3.52 & -2.80 \\
\hline $158-7-5$ & 61 & Pliocene marly chalk (pyrite, ash) & - & - & - & - & - & - & 3.46 & -1.25 \\
\hline $158-9-6$ & 80 & Late Miocene marly chalk (pyrite, ash) & 19.3 & 64 & 4.3 & 25.7 & 15.5 & $<0.05$ & 2.73 & -0.69 \\
\hline $158-15-4$ & 131 & yrite, ash) & - & - & - & - & - & - & 2.74 & -2.42 \\
\hline $158-21-5$ & 186 & Late Mioce & 19.4 & 64 & 3.7 & 36.2 & 19.0 & $<0.05$ & 1.53 & -1.29 \\
\hline $158-30-6$ & 269 & Middle Miocene marly chalk (pyrite, ash) & 19.1 & 64 & 3.4 & 40.0 & 20.0 & 0.1 & 0.93 & - \\
\hline $59-1-5$ & 7 & M- & 19. & 66 & & 17.5 & 0.8 & 0 & 2.20 & -11.10 \\
\hline $159-7-5$ & 61 & Early Mioc & 19 & 66 & 4.6 & - & 1.9 & $<0.05$ & 2.26 & -0.39 \\
\hline $159-12-5$ & 106 & Early Miocene marl ooze (Mn nodules) & 19.0 & 66 & 4.6 & - & 2.2 & $<0.05$ & 2.29 & -1.18 \\
\hline 16 & 3 & 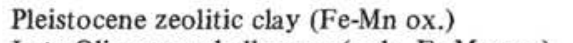 & 18.9 & 66 & 5.0 & - & 2.5 & $<0.05$ & 2.15 & -1.37 \\
\hline & 62 & ) & 1 & 9 & - & 19.3 & 2.8 & 05 & 2.01 & -0.89 \\
\hline $160-11-6$ & 98 & Early Oligocene chalk ooze (rads, Fe-Mn ox.) & 19.4 & 68 & 5.0 & 19.1 & 3.1 & $<0.05$ & 2.24 & - \\
\hline 16 & 3 & Early Miocene chalk ooze (rads, Fe-Mn ox.) & 19. & 6 & 4. & - & 1. & & 0 . & -5.69 \\
\hline $161-4-6$ & 35 & (rads, Fe-Mn ox.) & 19 & 6 & 5. & 18. & 0.8 & & & -8.20 \\
\hline $161 \mathrm{~A}-1-6$ & 71 & Late Oligocene chalk ooze (rads, diatoms) & 19.1 & 67 & 4.9 & 21.0 & 3.6 & & 2.84 & -2.50 \\
\hline $161-11-6$ & 98 & Middle Oligocene white chalk ooze & 19. & 64 & 4.8 & 22.1 & $<0.5$ & $<0.05$ & 2.33 & -1.17 \\
\hline $161 \mathrm{~A}-6-6$ & 172 & lk (rads) & 19.1 & 68 & 4.7 & 22.3 & 1.8 & $<0.05$ & 2.76 & -3.62 \\
\hline $161 \mathrm{~A}-11-4$ & 214 & Late Eocene calcareous radiolarite & 19.1 & 68 & 4.4 & 29.0 & $<0.5$ & $<0.05$ & 3.04 & -3.72 \\
\hline $162-1-6$ & 8 & $\mathrm{E}$ & 19. & 66 & 5 . & - & $<0.5$ & & 2.25 & -6.67 \\
\hline $162-8-6$ & 71 & Middle Eocene marl ooze (rads, Fe-Mn ox.) & 19.6 & 68 & 4.7 & 20.5 & 2.4 & & 1.70 & -0.70 \\
\hline $162-13-6$ & 116 & Middle Eocene marl ooze (rads, Fe-Mn ox.) & 19.5 & 67 & 4.5 & 24.0 & 3.1 & $<0.05$ & 2.76 & -0.31 \\
\hline $6-2$ & 138 & Middle Eocene marl (rads, chert) & 19.4 & 66 & 4.5 & 21.5 & 4.0 & $<0.05$ & 2.79 & -2.02 \\
\hline $163-2$ & 6 & 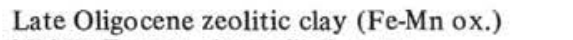 & 19 & 6 & 4 & 17 & 1.5 & $<0$ & 2.4 & -12.06 \\
\hline & 91 & & 19 & 6 & 4. & 22 & - & $<0.05$ & 1.5 & -5.99 \\
\hline $163 \mathrm{~A}-1-4$ & 145 & Unknown Age zeolitic clay (Fe-Mn ox.) & 18.8 & 66 & 5.2 & 22.6 & 2.4 & $<0.05$ & 1.10 & -2.64 \\
\hline $163-19-5$ & 205 & Upper Cretaceous nannofossil chalk (chert) & 19.1 & 66 & 4.7 & 17.4 & 1.3 & 1.8 & 2.59 & -3.83 \\
\hline $163-23-6$ & 242 & Upper Cretaceous nannofossil chalk (chert) & 18.5 & 64 & 3.7 & 23.2 & 3.7 & 0.8 & 0.66 & - \\
\hline
\end{tabular}

${ }^{\mathrm{a} D e p t h}$ in sediment column.

${ }^{b_{F}}$ From preliminary hole summaries. 